WŁodzimierz BĄK and TAdeusz Nadzieja (Opole)

\title{
EVOLUTION IN A MIGRATING POPULATION MODEL
}

Abstract. We consider a model of migrating population occupying a compact domain $\Omega$ in the plane. We assume the Malthusian growth of the population at each point $x \in \Omega$ and that the mobility of individuals depends on $x \in \Omega$. The evolution of the probability density $u(x, t)$ that a randomly chosen individual occupies $x \in \Omega$ at time $t$ is described by the nonlocal linear equation $u_{t}=\int_{\Omega} \varphi(y) u(y, t) d y-\varphi(x) u(x, t)$, where $\varphi(x)$ is a given function characterizing the mobility of individuals living at $x$. We show that the asymptotic behaviour of $u(x, t)$ as $t \rightarrow \infty$ depends on the properties of $\varphi$ in the vicinity of its zeros.

1. Introduction. Evolution problems of the form

$$
\partial_{t} u(x, t)=G(u, x, t)-u(x, t) L u(x, t),
$$

where $G$ is in general a nonlinear operator, which depends on $u$ in nonlocal way, and $L$ is a linear operator, have been considered in many papers; see e.g. [1], [2], [3], [5], [6] and the references therein.

For example, the equation

$$
\partial_{t} u(x, t)=\int_{\mathbb{R}^{n}} J(x-y) u(y, t) d y-u(x, t)
$$

has been applied in [1] to describe the evolution of the density $u(x, t)$ of a population. Here the function $J(x-y)$ is interpreted as the migration probability from location $y$ to location $x$ and $-u(x, t)$ is the rate at which individuals leave $x$ to move to any other site.

The authors of [5] give many other examples of biological phenomena which can be modelled by equation (1).

In this paper we will consider a particular form of equation (1). A population of density $m(x, t)$ occupies a compact domain $\Omega \subset \mathbb{R}^{2}$ so that

2010 Mathematics Subject Classification: 35R09, 92D25.

Key words and phrases: nonlocal differential equation, evolution of population density. 
$\int_{\omega} m(x, t) d x$ is the mass of the population in a subdomain $\omega \subset \Omega$ at time $t$. Assume that the density of newborn individuals is proportional to $m(x, t)$ (the Malthusian law of growth of population), and moreover, that the individuals living at $x$ can move to other points in $\Omega$. Let $m_{o}(x, t)$ be the density of outgoing individuals, and $m_{i}(x, t)$ the density of incoming individuals. The mobility of individuals living at $x$ is characterized by a nonnegative, continuous function $\varphi$ on $\Omega, 0 \leq \varphi(x) \leq 1$, i.e. the density has the form $m_{o}(x, t)=\varphi(x) m(x, t)$. Assume that $m_{i}(x, t)$ depends only on $t$, i.e. individuals can move from a point $x$ in $\Omega$ to another one with probability independent of the destination. This assumption and the fact that the mass of outgoing individuals equals the mass of incoming individuals lead to

$$
\int_{\Omega} m_{o}(x, t) d x=\int_{\Omega} \varphi(x) m(x, t) d x=\int_{\Omega} m_{i}(t) d x=m_{i}(t)|\Omega|,
$$

where $|\Omega|$ denotes the volume of the domain $\Omega$. Below we assume, for simplicity, that $|\Omega|=1$. Hence $m_{i}(t)=\int_{\Omega} \varphi(y) m(y, t) d y$.

The change of $m(x, t)$ during the time interval $\Delta t$ depends on:

(i) the mass of newborn individuals, which according to the Malthusian law equals $\operatorname{am}(x, t) \Delta t$, where $a>0$ is a constant;

(ii) the growth of the mass of incoming individuals, which is $m_{i}(t) \Delta t$;

(iii) the growth of the mass of outgoing individuals, which is $m_{o}(x, t) \Delta t$.

The above assumptions lead to the following continuity equation:

$$
\partial_{t} m(x, t)=\operatorname{am}(x, t)+\int_{\Omega} \varphi(y) m(y, t) d y-\varphi(x) m(x, t) .
$$

Equation (2) is supplemented with the initial condition

$$
m(x, 0)=m_{0}(x),
$$

where $m_{0}(x) \geq 0$ is a given initial density. Integrating (2) over $\Omega$ we get an equation for the total mass $M(t)$ of the population, $M^{\prime}(t)=a M(t)$. Hence $M(t)=M_{0} \exp (a t)$, where $M_{0}=\int_{\Omega} m_{0}(x) d x$.

The function $u(x, t)=m(x, t) / M(t)$ is the probability density that a randomly chosen individual at time $t$ lives at a point $x \in \Omega$. The evolution of $u(x, t)$ is described by the linear nonlocal problem

$$
\begin{aligned}
\partial_{t} u(x, t) & =\int_{\Omega} \varphi(y) u(y, t) d y-\varphi(x) u(x, t)=: A u(t)-\varphi(x) u(x, t), \\
u(x, 0) & =u_{0}(x),
\end{aligned}
$$

where $u_{0}(x)=m_{0}(x) / M_{0}$. Problem (4)-(5) can be considered for $\Omega \subset \mathbb{R}^{n}$ with $n>2$, but this does not lead to more interesting mathematical phenomena. Indeed, (4) is a family of ordinary differential equations indexed by the parameter $x$ and the dimension of the space of parameters is not impor- 
tant for our considerations. The choice $n=2$ is motivated by a biological interpretation of the problem.

By a solution of problem (4)-(5) we mean a continuous function of $x$ and $t$ on $\Omega \times[0, T]$, differentiable with respect to $t$, which satisfies (4) pointwise on $\Omega \times[0, T]$ and satisfies the initial condition (5).

For a fixed $x, u(x, t)$ as a function of $t$ satisfies a linear differential equation with the initial data $u_{0}(x)$, hence by the variation of parameters formula

$$
u(x, t)=u_{0}(x) e^{-\varphi(x) t}+e^{-\varphi(x) t} \int_{0}^{t} e^{\varphi(x) s} A u(s) d s .
$$

Equation (6) is the integral form of the differential problem (4)-(5), which is quite convenient to prove the existence of solution of (4)-(5) as well as to study its properties.

Lemma 1.1. If $\lim _{t \rightarrow \infty} A u(t)=0$ and $\varphi(x) \geq a>0$ on $B \subset \Omega$, then $u(x, t) \rightarrow 0$ as $t \rightarrow \infty$ uniformly on $B$.

Proof. We rewrite (6) in the form

$$
u(x, t)=u_{0}(x) e^{-\varphi(x) t}+e^{-\varphi(x) t} \int_{0}^{T} e^{\varphi(x) s} A u(s) d s+e^{-\varphi(x) t} \int_{T}^{t} e^{\varphi(x) s} A u(s) d s .
$$

For a given $\varepsilon>0$ we choose $T$ such that $A u(t)<\epsilon$ for $t>T$. Then for $x \in B$, we obtain

$$
u(x, t) \leq u_{0}(x) e^{-a t}+e^{-a t} \int_{0}^{T} e^{\varphi(x) s} A u(s) d s+\varepsilon \frac{1-e^{T-t}}{a} .
$$

Letting $t \rightarrow \infty$, we see that the solution $u(x, t)$ converges to 0 uniformly on $B$.

2. Existence of solution and its properties. Problem (4)-(5) features properties characteristic for diffusion problems. The reason is that equation (4), after some transformation of time, becomes the nonlocal diffusion equation related to the functional (see [4] for the definition of nonlocal diffusion)

$$
\mathcal{F}(v)=\iint_{\Omega}(\varphi(x) v(x)-\varphi(y) v(y))^{2} d x d y .
$$

One of the characteristic properties of diffusion problems is the existence of an entropy or, in other words, of a Lyapunov functional.

Property 1. The square of the $L^{2}$-norm of the solution of (4)-(5) with respect to the measure $\varphi(x) d x, \mathcal{L} u(t)=\int_{\Omega} u^{2}(x, t) \varphi(x) d x$, is a Lyapunov functional for that problem. 
Proof. Multiplying (4) by $\varphi u$ we get

$$
\frac{1}{2} \frac{d}{d t} \varphi\left(u^{2}\right)=\varphi u A u-\varphi^{2} u^{2} .
$$

Integrating (7) over $\Omega$, and using the Jensen inequality, we obtain

$$
\begin{aligned}
\frac{1}{2}(\mathcal{L} u)^{\prime}(t) & =\frac{1}{2} \frac{d}{d t}\left(\int_{\Omega} \varphi(x) u^{2}(x, t) d x\right) \\
& =(A u(t))^{2}-\int_{\Omega} \varphi^{2}(x) u^{2}(x, t) d x \leq 0 .
\end{aligned}
$$

PROPERTY 2. The unique solution with homogeneous initial data is $u(x, t) \equiv 0$.

Proof. We have $\mathcal{L} u(t) \equiv 0$, so the Lebesgue measure of the intersection of the supports of $u(\cdot, t)$ and $\varphi$ is zero. Hence (4) takes the form $\partial_{t} u(x, t)=$ $-\varphi(x) u(x, t)$, which implies that $u(x, t)=u_{0}(x) e^{-\varphi(x) t} \equiv 0$.

The next property says that, under some assumption, the solution $u$ becomes instantaneously positive, so $u$ diffuses at infinite speed.

PROPERTY 3. If the intersection of the supports of $u_{0}$ and $\varphi$ has a positive Lebesgue measure, then $u(x, t)>0$ for all $t>0$.

Proof. Our asumption implies that $A u(t)>0$ for small $t$. Now the positivity of the solution is a consequence of the integral equation (6) satisfied by any solution of (4)-(5).

If the assumption of Property 3 is not satisfied, we can write the solution of our problem in an explicit form.

PROPERTY 4. If the supports of $u_{0}$ and $\varphi$ do not meet in a set of positive Lebesgue measure, then $u(x, t)=u_{0}(x)$ is the unique solution of our problem.

Proof. It is easy to check that $u(x, t)=u_{0}(x)$ is a solution. Assume that there exists another solution $v$. Because our problem is linear, $w=u-v$ is a solution of (4) with homogeneous initial data. Using Property 2 we have $w \equiv 0$, and so $u(x, t)=v(x, t)$.

The problem (4)-(5) describes the evolution of density, hence its solution must satisfy

Property 5. The solution $u(x, t)$ is nonnegative and $\int_{\Omega} u(x, t) d x=1$.

Proof. The conservation of the $L^{1}$-norm is obvious, it is enough to integrate (4) over $\Omega$. The nonnegativity of $u$ is a consequence of Properties 3 and 4 .

For the proof of existence of such a solution we use the Banach contraction principle. 
TheOREM 2.1. There exists a unique solution of problem (4)-(5).

Proof. It follows from Property 5 that it is enough to look for nonnegative solutions. For fixed $T>0$ and $\lambda>0$ we define

$$
X=\left\{u:[0, T] \rightarrow C^{0}(\Omega): u \geq 0, u(0)=u_{0}\right\} .
$$

The set $X$ equipped with the distance function

$$
d_{\lambda}(u, v)=\sup _{t \in[0, T]} e^{-\lambda t}\|u(t)-v(t)\|_{C^{0}(\Omega)}
$$

is a complete metric space. The operator $W$ defined on $\left(X, d_{\lambda}\right)$ by

$$
W v(t)(x)=u_{0}(x) e^{-\varphi(x) t}+e^{-\varphi(x) t} \int_{0}^{t} e^{\varphi(x) s} \int_{\Omega} \varphi(x) v(s)(y) d y d s
$$

is a contraction on $X$ for $\lambda>1$. In fact, we have

$$
\begin{aligned}
d_{\lambda}(W u, W v) & =\sup _{t \in[0, T]} e^{-\lambda t}\|W u(t)(x)-W v(t)(x)\|_{C^{0}(\Omega)} \\
& \leq \sup _{t \in[0, T]}\left\|e^{-(\lambda+\varphi(x)) t} \int_{0}^{t} e^{\varphi(x) s} \int_{\Omega} e^{\lambda s} e^{-\lambda s}|u(s)(y)-v(s)(y)| d y d s\right\|_{C^{0}(\Omega)} \\
& \leq \sup _{t \in[0, T]}\left\|e^{-(\lambda+\varphi(x)) t} \int_{0}^{t} e^{\varphi(x) s} \int_{\Omega} e^{\lambda s} d_{\lambda}(u, v) d y d s\right\|_{C^{0}(\Omega)} \\
& =d_{\lambda}(u, v) \sup _{t \in[0, T]}\left\|e^{-(\lambda+\varphi(x)) t} \int_{0}^{t} e^{(\lambda+\varphi(x)) s} d s\right\|_{C^{0}(\Omega)} \leq \frac{1}{\lambda} d_{\lambda}(u, v) . \\
& =d_{\lambda}(u, v) \sup _{t \in[0, T]}\left\|\frac{1-e^{-(\lambda+\varphi(x)) t}}{\lambda+\varphi(x)}\right\|_{C^{0}(\Omega)}
\end{aligned}
$$

3. Asymptotic behaviour of solutions. The asymptotic properties of solutions of (4)-(5) depend on the behaviour of the function $\varphi$ in the vicinity of the set of its zeros, $B=\{x \in \Omega: \varphi(x)=0\}$. If $\varphi>0$, the solution tends as $t \rightarrow \infty$ uniformly to the unique stationary solution, thus we may say that the steady state coincides with this stationary solution. If $B \neq \emptyset$, the situation is more complicated. The steady state is a measure with a density or a singular measure, depending on the properties of $1 / \varphi$.

This kind of asymptotic behaviour is characteristic for semigroups of Markov operators for which the Foguel alternative is satisfied, i.e. sweeping occurs or the semigroup is asymptotically stable (for a detailed presentation see [7] and the references therein).

First, we consider the case $B=\emptyset$, i.e. $\varphi>0$ on $\Omega$. The stationary solution $U$ satisfies $A U(x)=\varphi(x) U(x)$, hence $U(x)=A(U) / \varphi(x)$. Here $A(U)$ is a constant, which depends on the unknown density $U$. We have $\int_{\Omega} U(x) d x=1$, 
so $A(U)=\left(\int_{\Omega}(\varphi(x))^{-1} d x\right)^{-1}$. This implies that the stationary solution is of the form

$$
U(x)=\left(\int_{\Omega} \frac{1}{\varphi(y)} d y\right)^{-1} \frac{1}{\varphi(x)} .
$$

THEOREM 3.1. If $\varphi(x)>0$ on $\Omega$, then the solution of (4)-(5) tends uniformly to the stationary solution $U$ defined in (9).

Proof. The function $v(x, t)=u(x, t)-U(x)$ satisfies equation (4) and $\int_{\Omega} v(x, t) d x=0$. Using the Cauchy inequality we get

$$
A v(t) \leq\left(\int_{\Omega} \varphi(x) d x\right)^{1 / 2}(\mathcal{L} v(t))^{1 / 2} .
$$

The $L^{2}$-norm of $v$ satisfies

$$
\begin{aligned}
\left(\|v\|_{2}^{2}\right)_{t} & =\frac{d}{d t}\left(\int_{\Omega} v^{2} d x\right)=2 \int_{\Omega} v(x, t) d x A v(t)-2 \int_{\Omega} v^{2}(x, t) \varphi(x) d x \\
& =-2 \mathcal{L} v(t) .
\end{aligned}
$$

Now, it follows from (11) that $\mathcal{L} v$ tends to 0 as $t \rightarrow \infty$. Hence 10 implies that $A v(t) \rightarrow 0$ as $t \rightarrow \infty$. Using Lemma 1.1, we get the uniform convergence of solutions to the steady state $U$.

If $B \neq \emptyset$ and $\int_{\Omega} 1 / \varphi(x) d x<\infty$, we define $U(x)$ by 9 for $x \notin B$ and $U(x)=\infty$ on $B$, and we call such a function the steady state.

TheOREM 3.2. If $\int_{\Omega} 1 / \varphi(x) d x<\infty$, then the solution of (4)-(5) converges to the steady state as $t \rightarrow \infty$ uniformly on each compact subset of $\Omega \backslash B$.

Proof. Again we consider the difference $v(x, t):=u(x, t)-U(x)$. Evidently $v$ satisfies $(4)$ on $\Omega \backslash B$ and $\int_{\Omega} v(x, t) d x=0$. We cannot, as before, differentiate the $L^{2}$-norm of $v$ (it is not even clear if $v$ is in $L^{2}(\Omega)$ or not).

However, we can still use the Lyapunov functional which is well defined for $v$, and inequality (10) holds. We show that $A v(t) \rightarrow 0$ as $t \rightarrow \infty$. First, we prove that there exists a sequence $t_{n} \rightarrow \infty$ such that $A v\left(t_{n}\right) \rightarrow 0$. Suppose that $A v(t)>a>0$ for all $t>0$. Then

$$
\partial_{t} v(x, t) \geq a-\varphi(x) v(x, t)
$$

and therefore

$$
v(x, t) \geq \frac{a}{\varphi(x)}-\left(\frac{a}{\varphi(x)}-v_{0}(x)\right) e^{-\varphi(x) t} .
$$

For each neighbourhood $K_{r}=\{x \in \Omega$ : $\operatorname{dist}(x, B)<r\}$ of $B$, and for suf- 
ficiently large $t$, we have

$$
\int_{\Omega \backslash K_{r}} v(x, t) d x \geq \frac{1}{2} \int_{\Omega \backslash K_{r}} \frac{a}{\varphi(x)} d x .
$$

Thus, for sufficiently small $r$ we get

$$
\begin{aligned}
\int_{K_{r}} v(x, t) d x & =\int_{K_{r}} u(x, t) d x-\int_{K_{r}} U(x) d x \geq-\int_{K_{r}} U(x) d x \\
& >-\frac{1}{4} \int_{\Omega \backslash K_{r}} \frac{a}{\varphi(x)} d x .
\end{aligned}
$$

Inequalities 14 - 15 imply $\int_{\Omega} v(x, t) d x>0$ for sufficiently large $t$, which leads to a contradiction.

Suppose that there exists a sequence $\bar{t}_{n} \rightarrow \infty$ such that $A v\left(\bar{t}_{n}\right)>\bar{a}$ for some $\bar{a}>0$. Note that

$$
(A v)^{\prime}(t)=\int_{\Omega} \varphi(x) d x A v(t)-\int_{\Omega} v(x, t) \varphi^{2}(x) d x .
$$

It follows from 10 that $A v(t)$ is bounded. Moreover we have

$$
\left|\int_{\Omega} v(x, t) \varphi(x) d x\right| \leq \max \varphi^{2}(x) \int_{\Omega}|u(x, t)-U(x)| d x \leq 2 \max \varphi^{2}(x)
$$

and hence $(A v)^{\prime}(t)$ is bounded on $\mathbb{R}^{+}$.

To show that $A v(t) \rightarrow 0$, it is enough to prove that $\mathcal{L} v(t) \rightarrow 0$. Assume to the contrary that $\mathcal{L} v(t)>b>0$ for all $t>0$ and some $b$.

Note that

$$
\frac{d}{d t}\left(\int_{\Omega} u^{2}(x, t) d x\right)=A v(t)-\mathcal{L} v(t)
$$

As shown above, there exists $t_{n} \rightarrow \infty$ such that $A v\left(t_{n}\right) \rightarrow 0$, and the derivative of $A v(t)$ is bounded. Hence for some $\delta>0$ the intervals $\Delta_{n}:=$ $\left(t_{n}-\delta, t_{n}+\delta\right)$ are such that on $\Delta_{n}$,

$$
\frac{d}{d t}\left(\int_{\Omega} u^{2}(x, t) d x\right)<-\frac{1}{2} b .
$$

This implies that $\int_{\Omega} u^{2}(x, t) d x$ becomes negative in finite time, which is absurd. In this way we proved that $\mathcal{L} v(t) \rightarrow 0$, so $A v(t) \rightarrow 0$.

If $1 / \varphi$ is not integrable, we have only partial results about the asymptotic behaviour of solutions. If $B$ is a single point, $B=\left\{x_{0}\right\}$, and $1 / \varphi$ is not integrable, we call the measure $\delta_{x_{0}}$ the stationary state. This is justified by the next theorem. 
THEOREM 3.3. If $B=\left\{x_{0}\right\}$ and $1 / \varphi$ is not integrable, then the solution of problem (4)-(5) tends to $\delta_{x_{0}}$ as $t \rightarrow \infty$ in the sense of weak convergence of measures.

Proof. We show that $A u(t) \rightarrow 0$ as $t \rightarrow \infty$. First, we prove that there exists a sequence $t_{n}$ tending to $\infty$ such that $A u\left(t_{n}\right) \rightarrow 0$. Assume that $A u(t) \geq a>0$, hence

$$
u(x, t) \geq \frac{a}{\varphi(x)}-\left(\frac{a}{\varphi(x)}-u_{0}(x)\right) e^{-\varphi(x) t} .
$$

For $\delta>0$ we define $E_{\delta}=\left\{x \in \Omega:\left\|x-x_{0}\right\|>\delta\right\}$. Now we choose $\delta>0$ such that $\frac{1}{2} a \int_{E_{\delta}} 1 / \varphi(x) d x>2$. For each sufficiently large $t$,

$$
\left(\frac{a}{\varphi(x)}-u_{0}(x)\right) e^{-\varphi(x) t}<1,
$$

so we get $\int_{E_{\delta}} u(x, t) d x>1$, a contradiction. Note that

$$
(A u)^{\prime}(t)=A u(t) \int_{\Omega} \varphi(x) d x-\mathcal{L} u(t),
$$

and the derivative $(A u)^{\prime}(t)$ is bounded. To prove that $\mathcal{L} u(t)$ tends to 0 , we proceed as in the preceding proof. Having this convergence and using (10) (with $u$ instead of $v$ ), we know that $A u(t) \rightarrow 0$ and Lemma 1.1 implies that $u(\cdot, t)$ goes to 0 uniformly on each compact subset of $\Omega \backslash\left\{x_{0}\right\}$.

Acknowledgements. We are grateful to Piotr Biler and Andrzej Spakowski for interesting conversations during the preparation of this paper.

\section{References}

[1] F. Andreu, J. M. Mazón, J. D. Rossi and J. Toledo, The Neumann problem for nonlocal nonlinear diffusion equations, J. Evol. Equations 8 (2008), 189-215.

[2] C. Carrillo and P. Fife, Spatial effects in discrete generation population models, J. Math. Biol. 50 (2005), 161-188.

[3] L. Desvillettes, P.-E. Jabin, S. Mischler and G. Raoul, On selection dynamics for continuous structured populations, Comm. Math. Sci. 6 (2008), 729-747.

[4] P. Fife, Some Nonclassical Trends in Parabolic and Parabolic-like Evolutions, in: Trends in Nonlinear Analysis, Springer, Berlin, 2003, 153-191.

[5] M. Lachowicz and D. Wrzosek, Nonlocal bilinear equations. Equilibrium solutions and diffusive limit, Math. Models Methods Appl. Sci. 11 (2001), 1393-1409.

[6] G. Raoul, Long time evolution of populations under selection and vanishing mutations, Acta Appl. Math. 114 (2011), 1-14.

[7] R. Rudnicki, K. Pichór and M. Tyran-Kamińska, Markov semigroups and their applications, in: Dynamics of Dissipation, P. Garbaczewski and R. Olkiewicz (eds.), Lecture Notes in Phys. 597, Springer, Berlin, 2002, 215-238. 
Włodzimierz Bąk, Tadeusz Nadzieja

Instytut Matematyki i Informatyki

Uniwersytet Opolski

Oleska 48

45-052 Opole, Poland

E-mail: wbak@math.uni.opole.pl tnadzieja@math.uni.opole.pl

Received on 2.2.2011;

revised version on 26.6.2012 
